\title{
The XMM-Newton view of IRAS 09104+4109: evidence for a changing-look Type 2 quasar?
}

\author{
E. Piconcelli ${ }^{1}$, F. Fiore ${ }^{1}$, F. Nicastro ${ }^{1,2}$, S. Mathur ${ }^{3}$, M. Brusa ${ }^{4}$, A. Comastri ${ }^{5}$, and S. Puccetti ${ }^{1,6}$ \\ 1 Osservatorio Astronomico di Roma (INAF), via Frascati 33, 00040 Monteporzio Catone (Roma), Italy \\ e-mail: piconcelli@oa-roma.inaf.it \\ 2 Harvard-Smithsonian Center for Astrophysics, 60 Garden Street, Cambridge, MA 02138, USA \\ Ohio State University, 140 West 18th Avenue, Columbus, OH 43210, USA \\ 4 Max Planck Institut für Extraterrestrische Physik, Postfach 1312, 85741 Garching bei München, Germany \\ 5 Osservatorio Astronomico di Bologna (INAF), via Ranzani 1, 40127 Bologna, Italy \\ 6 ASI Science Data Center, ESRIN, via G. Galilei, 00044 Frascati, Italy
}

Received 11 April 2007 / Accepted 6 July 2007

\section{ABSTRACT}

\begin{abstract}
Aims. We report on a $14 \mathrm{ks}$ XMM-Newton observation of the hyperluminous infrared galaxy IRAS 09104+4109, which harbors a type 2 quasar in its nucleus. Our analysis was aimed at studying the properties of the absorbing matter and the Fe $\mathrm{K}$ complex at 6-7 keV in this source.

Methods. We analyzed the spectroscopic data from the $P N$ and the $M O S$ cameras in the $0.4-10 \mathrm{keV}$ band. We also used an archival BeppoSAX 1-50 keV observation of IRAS 09104+4109 to investigate possible variations of the quasar emission.

Results. The X-ray emission in the EPIC band is dominated by the intra-cluster medium thermal emission. We found that the quasar contributes $\sim 35 \%$ of the total flux in the $2-10 \mathrm{keV}$ band. Both a transmission- (through a Compton-thin absorber with a Compton optical depth of $\tau_{\mathrm{C}} \sim 0.3$, i.e. $N_{\mathrm{H}} \sim 5 \times 10^{23} \mathrm{~cm}^{-2}$ ) and a reflection-dominated $\left(\tau_{\mathrm{C}}>1\right)$ model provide an excellent fit to the quasar continuum emission. However, the value measured for the $E W$ of $\mathrm{Fe} \mathrm{K} \alpha$ emission line is only marginally consistent with the presence of a Compton-thick absorber in a reflection-dominated scenario, which had been suggested by a previous, marginal (i.e. $2.5 \sigma)$ detection with the hard X-ray (15-50 keV), non-imaging BeppoSAX/PDS instrument. Moreover, the value of luminosity in the 2-10 keV band measured by the transmission-dominated model is fully consistent with that expected on the basis of the bolometric luminosity of IRAS $09104+4109$. From the analysis of the XMM-Newton data we therefore suggest the possibility that the absorber along the line of sight to the nucleus of IRAS 09104+4109 is Compton-thin. Alternatively, the absorber column density could have changed from Compton-thick to -thin in the five years elapsed between the observations. If this is the case, then IRAS 09104+4109 is the first "changing-look" quasar ever detected.
\end{abstract}

Key words. galaxies: individual: IRAS 09104+4109 - galaxies: active - galaxies: nuclei - X-ray: galaxies

\section{Introduction}

IRAS $09104+4109$ is one the most powerful objects in the $z \lesssim 0.5$ Universe. It is a hyperluminous infrared $\mathrm{cD}$ galaxy of IR luminosity $>10^{13} L_{\odot}$ at $z=0.442$, residing in the core of a rich cluster of galaxies (Kleinmann et al. 1988). Its optical spectrum shows only narrow emission lines, but broad Balmer and $\mathrm{Mg}$ II emission lines were observed in the polarized light (Hines \& Wills 1993; Tran et al. 2000). These pieces of evidence have led to the conclusion that a dust-enshrouded type 2 quasar lies in the nucleus of IRAS $09104+4109$.

The X-ray observations of IRAS $09104+4109$ collected so far lend support to this suggestion. Franceschini et al. (2000; F00) reported on the analysis of a BeppoSAX observation of this source. They found that the X-ray spectrum below $10 \mathrm{keV}$ is dominated by the intra-cluster medium (ICM) thermal $(k T \sim$ $5.5 \mathrm{keV}$ ) emission. The detection of a weak signal in the $15-$ $60 \mathrm{keV}$ band with the PDS instrument was interpreted by F00 as the primary emission of the buried quasar, which emerges from an absorbing screen of $N_{\mathrm{H}} \gtrsim 5 \times 10^{24} \mathrm{~cm}^{-2}$. They also reported on the marginal detection of a neutral $\mathrm{Fe} \mathrm{K} \alpha$ emission line with an equivalent width $(E W)$ of $\sim 1-2 \mathrm{keV}$, consistent with a reflection-dominated scenario for the quasar emission in the
2-10 keV band. This evidence makes this source the best example of a Compton-thick type 2 quasar found to date. Exploiting the high spatial resolution of Chandra, Iwasawa et al. (2001, I01 hereafter) were able to analyze the spectral data of the central source embedded in the extended ICM emission. Although limited by the low statistics ( 200 counts in the range $0.6-7 \mathrm{keV}$ ), these data allowed to confirm the presence of a heavily obscured quasar and, by the comparison with the BeppoSAX PDS flux, I01 concluded that the Chandra spectrum is also reflectiondominated.

\section{XMM-Newton observation and data reduction}

IRAS 09104+4109 was observed with XMM-Newton (Jansen et al. 2001, and references therein) on April 27, 2003 for $\sim 14 \mathrm{ks}$. The EPIC PN and MOS observations were carried out in the full frame mode using the Medium filter. XMM-Newton data were processed with SAS v6.5. We used the EPCHAIN and EMCHAIN tasks for processing the raw $P N$ and MOS data files, respectively, to generate the relative linearized event files. X-ray events corresponding to patterns 0-12(0-4) for the $\operatorname{MOS}(P N)$ cameras were selected. Hot and bad pixels were removed. The event lists were furthermore filtered to ignore 
Table 1. Best-fit spectral parameters of the EPIC spectrum. See Sect. 3 for details. Column: (1) spectral model; (2) temperature of the ICM "cool core" component (keV); (3) ICM metallicity; (4) $2-10 \mathrm{keV}$ flux of the quasar component $\left(10^{-13} \mathrm{erg} \mathrm{cm}^{-2} \mathrm{~s}^{-1}\right.$ ); (5) $2-10 \mathrm{keV}$ luminosity of the quasar component $\left(10^{44} \mathrm{erg} \mathrm{s}^{-1}\right)$; (6) column density of the absorber $\left(10^{23} \mathrm{~cm}^{-2}\right)$; (7) energy of the $\mathrm{Fe} \mathrm{K} \alpha$ line (keV); (8) intensity of the Fe K $\alpha$ line $\left(10^{-6} \mathrm{ph} / \mathrm{cm}^{2} / \mathrm{s}\right) ;(9) E W$ of the $\mathrm{Fe} \mathrm{K} \alpha$ line $(\mathrm{eV}) ;(10)$ reduced $\chi^{2}$ and number of degrees of freedom.

\begin{tabular}{cccccccccc}
\hline \hline $\begin{array}{c}\text { Model }^{a} \\
\text { (1) }\end{array}$ & $k T$ & $\begin{array}{c}Z / Z_{\odot} \\
(2)\end{array}$ & $\begin{array}{c}F_{2-10} \\
(3)\end{array}$ & $\begin{array}{c}L_{2-10} \\
(4)\end{array}$ & $\begin{array}{c}N_{\mathrm{H}} \\
(5)\end{array}$ & $\begin{array}{c}E_{\mathrm{K} \alpha} \\
(6)\end{array}$ & $\begin{array}{c}I_{\mathrm{K} \alpha} \\
(7)\end{array}$ & $\begin{array}{c}E W_{\mathrm{K} \alpha} \\
(8)\end{array}$ & $\begin{array}{c}\chi_{v}^{2} \text { (d.o.f.) } \\
(9)\end{array}$ \\
\hline (A) & $3.9_{-0.9}^{+0.8}$ & $0.47_{-0.10}^{+0.10}$ & 4.68 & 7.95 & $4.8_{-1.5}^{+2.6}$ & $6.38_{-0.06}^{+0.06}$ & $8.7_{-4.8}^{+6.2}$ & $390_{-212}^{+380, b}$ & $0.94(219)$ \\
(B) & $3.6_{-0.7}^{+0.8}$ & $0.48_{-0.10}^{+0.11}$ & 5.57 & $>2.05$ & - & $6.38_{-0.05}^{+0.05}$ & $4.0_{-1.9}^{+2.1}$ & $402_{-193}^{+212, c}$ & $0.96(220)$ \\
\hline
\end{tabular}

${ }^{a}$ (A) transmission model; (B) reflection model; ${ }^{b}$ absorption-corrected line against absorption-corrected continuum; ${ }^{c}$ with respect to the pure reflection component.
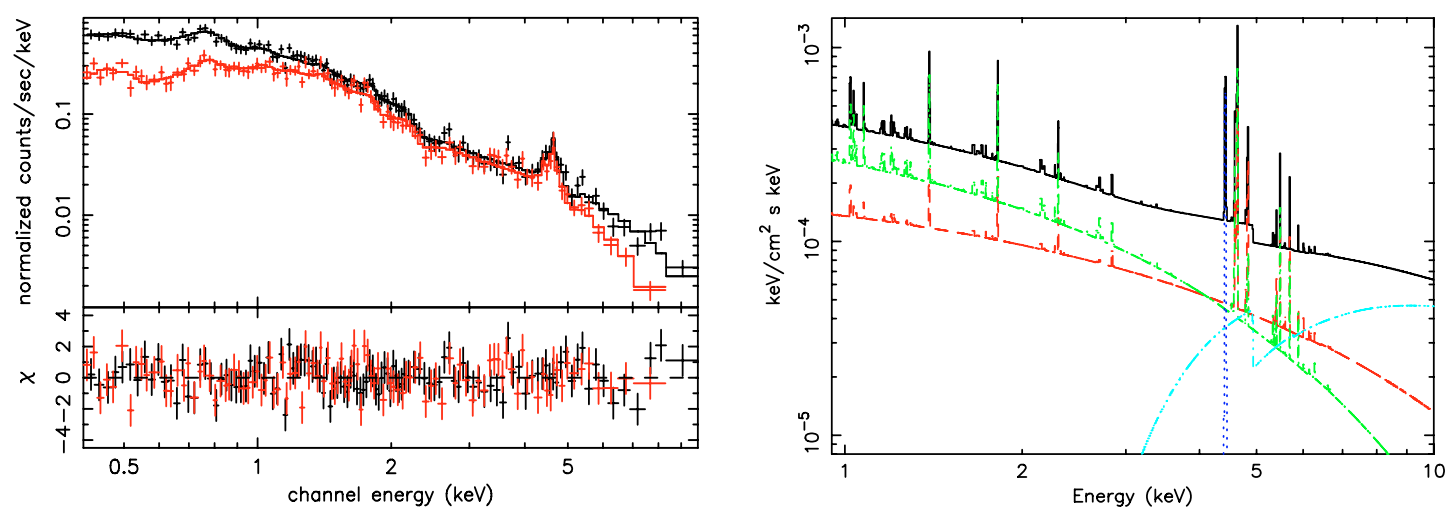

Fig. 1. a) Left: XMM-Newton $P N$ (top) and MOS (bottom) spectra of IRAS 09104+4109 when the transmission model is applied. The lower panel shows the deviations of the observed data from the model in unit of standard deviations. b) Right: best-fit model for the transmission scenario. The different spectral components are also plotted (e.g. Sect. 3 for further details).

periods of high background flaring according to the method presented in Piconcelli et al. (2004) based on the cumulative distribution function of background lightcurve count-rates. Final net exposures of $10.8,13.4$, and $13.4 \mathrm{ks}$ were obtained for $P N$, MOS1 and MOS2, respectively. The source photons were extracted for the $P N(M O S)$ camera from a circular region with a radius of $37(40)$ arcsec, while the background counts were estimated from a larger (i.e. $\sim 75$ arcsec radius) source-free region on the same chip. Appropriate response and ancillary files for all the $E P I C$ cameras were created using respectively RMFGEN and ARFGEN tasks in the SAS. Combined MOS1+MOS2 spectrum and response matrix were created.

Light-curves in the $0.2-2 \mathrm{keV}$ and $2-15 \mathrm{keV}$ band were extracted. Both light-curves are consistent with a constant flux level over the whole XMM-Newton exposure.

\section{Spectral modelling}

Both $P N$ and $M O S$ spectra were grouped to have a minimum of 20 counts per bin to allow the use of $\chi^{2}$ minimization technique and fitted simultaneously. Given the current calibration uncertainties, we discarded events below $0.4 \mathrm{keV}$ and above $10 \mathrm{keV}$. All fits were performed using the XSPEC package (v11.3) and included the Galactic column density value of $N_{\mathrm{H}}=1.81 \times 10^{20} \mathrm{~cm}^{-2}$ (Murphy et al. 1996). Best-fit parameter values are given in the source frame, unless otherwise specified. The quoted errors on the model parameters correspond to a $90 \%$ confidence level for one interesting parameter $\left(\Delta \chi^{2}=2.71\right.$; Avni 1976). A cosmology with $\left(\Omega_{\mathrm{M}}, \Omega_{\Lambda}\right)=(0.3,0.7)$ and a $H_{0}=70 \mathrm{~km} \mathrm{~s}^{-1} \mathrm{Mpc}^{-1}$ is assumed throughout.

The high-resolution ( $\lesssim 1$ arcsec) Chandra observation of IRAS $09104+4109$ presented by I01 has definitively established that the emission from the ICM dominates the X-ray spectrum below $10 \mathrm{keV}$. Furthermore IRAS $09104+4109$ is a massive cooling-flow cluster (Fabian \& Crawford 1995) showing a large radial temperature gradient, from $k T \approx 3.3 \mathrm{keV}$ in the bright, cool core up to $k T \approx 7.8 \mathrm{keV}$ at a distance of $200 \mathrm{kpc}$, i.e. $36 \operatorname{arcsec}$ (I01). For this reason, we initially fitted the combined $P N+M O S$ spectrum with a model consisting of two thermal plasma emission components (i.e. MEKAL in XSPEC) plus an absorbed power law. We fixed the temperature of one thermal component to $7.8 \mathrm{keV}$ in order not to underestimate the total ICM emission in the hard X-ray band. Since the photon index of the power law was loosely constrained $(\Gamma \approx 1.4 \pm 1)$ we fixed it to 1.8 , which is the average value typically observed for radio-quiet quasars (e.g. Piconcelli et al. 2005).

This model (transmission model hereafter) gave an excellent description of the EPIC data with a $\chi_{v}^{2}$ (d.o.f.) $=0.94(219)$ (see Fig. 1). The best-fit values of the spectral parameters are listed in Table 1 . The value of $\sim 4.8 \times 10^{23} \mathrm{~cm}^{-2}$ found for the column density translates in a Compton optical depth $\tau_{\mathrm{C}} \sim 0.3$ of the absorbing screen. The value of temperature $\left(k T=3.9_{-0.9}^{+0.8} \mathrm{keV}\right)$ in the cluster core and metallicity $\left(Z / Z_{\odot}=0.47_{-0.10}^{+0.10}\right)$ of the ICM are consistent with I01.

Works based on BeppoSAX and Chandra observations favored an interpretation of the spectrum of IRAS $09104+4109$ below $10 \mathrm{keV}$ in terms of reflection-dominated emission. We therefore replaced the absorbed power law in the transmission model with a Compton reflection component from neutral matter (i.e. PEXRAV model in XSPEC). For this spectral component, which is due to the reprocessing of the emission from the obscured primary X-ray source, we assumed a $\Gamma=1.8$ for the photon index of the incident power law, along with an inclination angle of $i=50 \mathrm{deg}$ (Tran et al. 2000) and solar metallicity for the reflector. This model (reflection model hereafter) yielded an equally good fit to the XMM-Newton spectrum with a final 

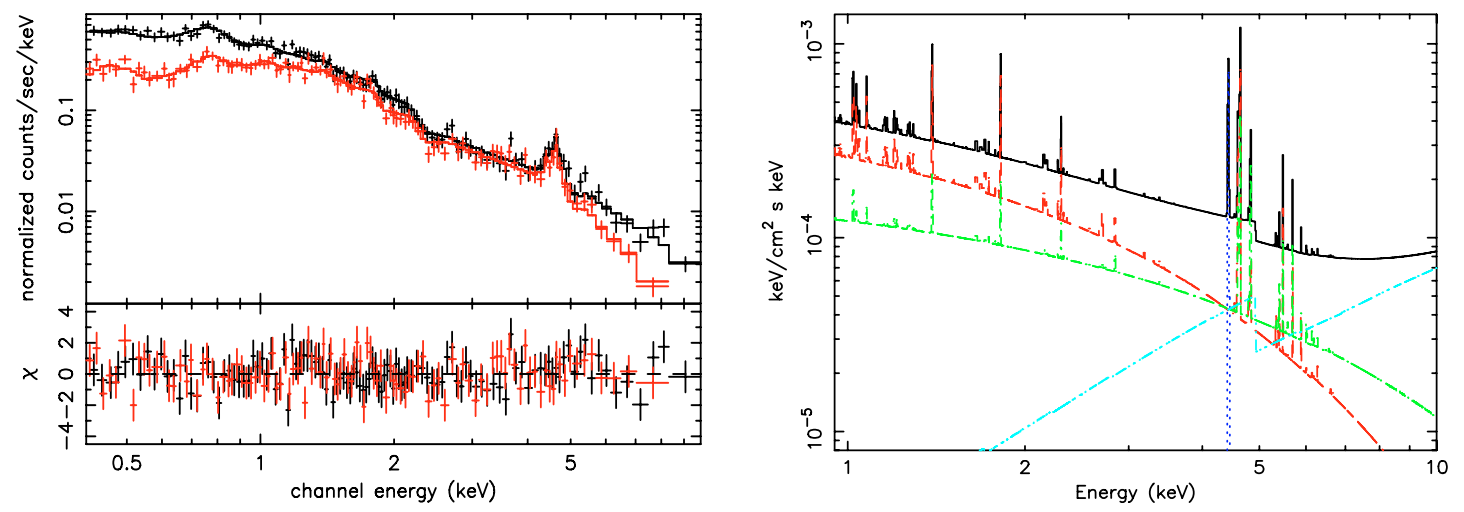

Fig. 2. The same as Fig. 1 for the reflection model.
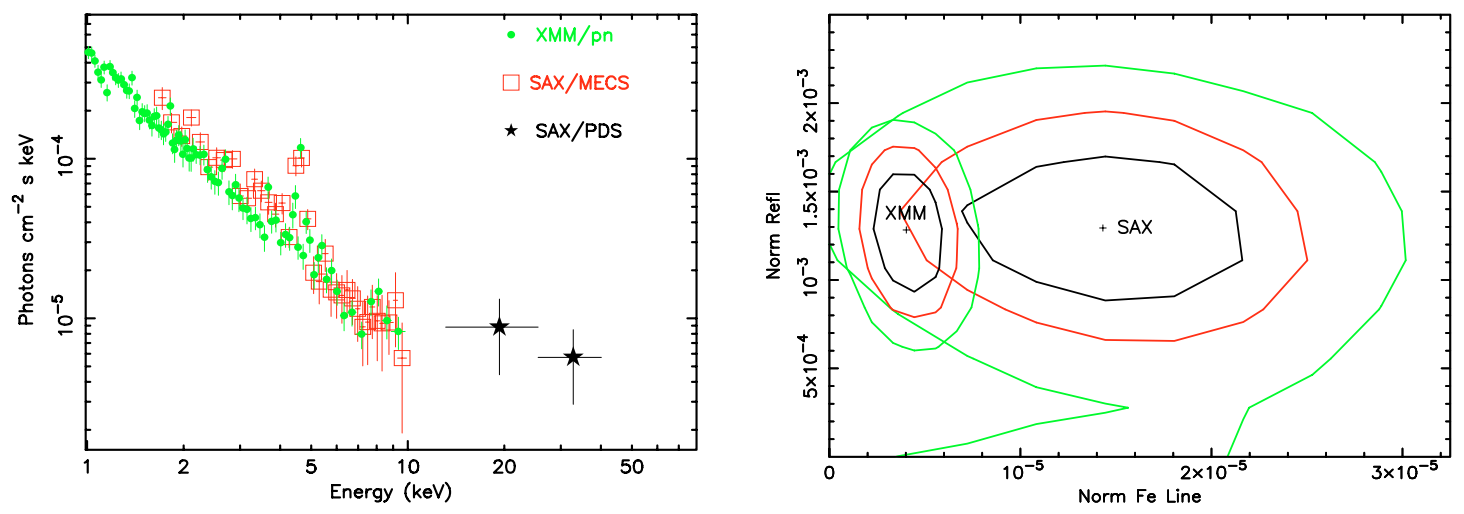

Fig. 3. a) Left: unfolded spectra for the 2003 XMM-Newton and 1998 BeppoSAX observations. b) Right: confidence contour plot showing the intensity of the $\mathrm{Fe} \mathrm{K} \alpha$ emission line (in units of $\mathrm{ph} / \mathrm{cm}^{2} / \mathrm{s}$ ) against the intensity (i.e. photon flux at $1 \mathrm{keV}$ ) of the reflection continuum (in units of $\mathrm{ph} / \mathrm{keV} / \mathrm{cm}^{2} / \mathrm{s}$ ) obtained using the XMM-Newton and BeppoSAX (MECS+PDS) data. The contours are at $68 \%, 90 \%$ and $99 \%$ confidence levels for two interesting parameters.

$\chi_{v}^{2}($ d.o.f. $)=0.96(220)$ (see Table 1 and Fig. 2). According to both spectral models, the contribution of the quasar component to the total flux in the $2-10 \mathrm{keV}$ band is $\approx 30-35 \%$.

As shown in Figs. 1a and 2a, there is a prominent line-like emission feature at $4-5 \mathrm{keV}$ (observer-frame) which is broader than the instrumental resolution at this energy and most likely due to a blend of lines associated with Fe K emission. This complex is partly accounted for by the strong FeXXV $\mathrm{K} \alpha$ emission line at $6.7 \mathrm{keV}$ and the FeXXVI $\mathrm{K} \alpha$ emission line at $6.97 \mathrm{keV}$ (which should be likely blended with a weak $\mathrm{Fe} \mathrm{K} \beta$ line at $7.06 \mathrm{keV}$ due to the reprocessing of the quasar continuum) from the two-temperature ICM. However, significant (at $>99 \%$ confidence level) positive residuals are still present. We modelled this excess with an unresolved Gaussian emission line at $6.38_{-0.06}^{+0.06} \mathrm{keV}$. This energy is consistent with a range of ionization states from FeI to FeXVI (Kallman et al. 2004), as typically observed in quasars (Jimenez-Bailon et al. 2005). In the "reflection-dominated" scenario we measure an equivalent width of the $\mathrm{Fe} \mathrm{K} \alpha$ line at $\sim 6.4 \mathrm{keV}$ of $E W_{\mathrm{K} \alpha}=402_{-193}^{+212} \mathrm{eV}$ (calculated with respect to the Compton reflection component). For the transmission model (i.e. assuming that the line and the continuum are both absorbed) we derive $E W_{\mathrm{K} \alpha}=390_{-212}^{+380} \mathrm{eV}$ (see Table 1).

\subsection{A comparison with broadband 1-50 keV BeppoSAX data}

To investigate on the possible year-timescale variability of the overall continuum spectral shape, we plot in Fig. 3a the unfolded $2003 P N$ and 1998 BeppoSAX MECS+PDS spectra. These data have been unfolded through the instrument response with respect to the best-fit model found by F00 (i.e. ICM thermal emission component + absorbed powerlaw + reflection + narrow Gaussian line at $6.4 \mathrm{keV}$; model sax 98 hereafter). As expected, the 1-10 keV XMM-Newton and BeppoSAX spectra have similar shape being dominated by the ICM emission. The different normalizations of the two spectra can be ascribed to the different source extraction regions. In particular, a fraction of ICM emission from the outskirt region of the cluster spreads outside the $P N / M O S$ extraction radius. Interestingly, the PDS data above $10 \mathrm{keV}$ appear to lie slightly above the extrapolation of the MECS/PN continuum level. We calculated a $20-30 \mathrm{keV}$ flux level of $F_{20-30} \sim 6.1 \times 10^{-13}$ and $\sim 1.3 \times 10^{-12} \mathrm{erg} \mathrm{cm}^{-2} \mathrm{~s}^{-1}$ for the transmission $^{1}$ and reflection model, respectively. The $20-30 \mathrm{keV}$ flux of IRAS 09104+4109 measured by BeppoSAX is $F_{20-30}=$ $2.55_{-1.56}^{+1.90} \times 10^{-12} \mathrm{erg} \mathrm{cm}^{-2} \mathrm{~s}^{-1}$, whereby the extrapolated $X M M-$ Newton flux in this band is fainter by a factor of $\sim 1.6-7.3$, if the transmission model is assumed. On the other hand, the 20$30 \mathrm{keV}$ flux estimated by reflection model is consistent with the BeppoSAX value.

Figure $3 \mathrm{~b}$ shows the iso- $\chi^{2}$ contour plot of the intensity of the Fe $\mathrm{K} \alpha$ emission line at $6.4 \mathrm{keV}\left(I_{\mathrm{K} \alpha}\right)$ versus the intensity of the reflection continuum for the 1998 BeppoSAX and 2003 XMMNewton observations. The XMM-Newton values were derived by the reflection model, while for the BeppoSAX data we employed the sax 98 model (temperature and abundance of the ICM

1 In the case of this model, we also added to the model a Compton reflection component with $R=1$ (where $R$ is the solid angle in units of $2 \pi$ subtended by the reflecting material) as commonly observed in the 10-50 keV AGN spectra (e.g. Risaliti 2002; Reeves et al. 2006). 
were permitted to vary within the $90 \%$ confidence interval measured for these parameters with XMM-Newton, e.g. Table 1). These measurements taken at different epochs are consistent within $3 \sigma$ errors, as expected if Compton hump and cold iron emission arise from distant material. Furthermore, the XMMNewton measurements are also compatible with the values of $I_{\mathrm{K} \alpha}=7.2_{-3.3}^{+6.1} \times 10^{-6}$ photons $\mathrm{cm}^{-2} \mathrm{~s}^{-1}$ and $E W_{\mathrm{K} \alpha}=1.1_{-0.5}^{+0.9} \mathrm{keV}$ reported by $\mathrm{I} 01$ on the basis of a 1999 Chandra observation of IRAS $09104+4109$. The mean values of $E W_{\mathrm{K} \alpha}$ were significantly larger, but the errors were also very large in the low $\mathrm{S} / \mathrm{N}$ BeppoSAX and Chandra spectra.

\section{Discussion}

The XMM-Newton observation presented here has confirmed the presence of a heavily absorbed quasar in the nucleus of the hyperluminous infrared galaxy IRAS 09104+4109. The EPIC energy range is dominated by the thermal emission of the ICM and we estimated that the quasar contributes approximately $\sim 30$ $35 \%$ of the total $2-10 \mathrm{keV}$ flux. We were able to accurately study the Fe K complex at $\sim 6-7 \mathrm{keV}$ in this source, modelling it with three distinct narrow emission lines from FeI-XVI K $\alpha$, FeXXV $\mathrm{K} \alpha$ and FeXXVI K $\alpha$ transition, respectively. The highly ionized $\mathrm{Fe} \mathrm{K}$ lines originate in the ICM, while the line from cold iron is likely due to reprocessing of the quasar continuum off the circumnuclear environment. The better quality of EPIC data has allowed to overcome the problems of limited spectral resolution and statistics of BeppoSAX data (e.g. F00), which prevented a correct estimate of the contribution from the different ions to the Fe emission complex.

The quasar emission can be equally well fitted by either a transmission or a reflection model. The former implies a Compton-thin absorber with $N_{\mathrm{H}} \approx 5 \times 10^{23} \mathrm{~cm}^{-2}$, while the latter suggests a scenario where the primary $\mathrm{X}$-ray continuum is blocked by a Compton-thick $\left(\tau_{\mathrm{C}} \gtrsim 1\right.$, i.e. $N_{\mathrm{H}} \gtrsim \sigma_{t}^{-1} \approx$ $1.6 \times 10^{24} \mathrm{~cm}^{-2}$ ) obscuring screen and the emission observed by $X M M-N e w t o n$ is due to indirect radiation scattered into the line of sight (it is generally assumed that the absorber and the reflector are the same material, i.e. the torus, e.g. Matt et al. 1996; Molendi et al. 2003). Using the present data these models are statistically indistinguishable. However, an $E W_{\mathrm{K} \alpha} \approx 400 \pm 200 \mathrm{eV}$ measured assuming the reflection model is significantly lower than expected for a truly "reflection-dominated" spectrum with $[\mathrm{Fe} / \mathrm{H}]=0$. In fact, the most prominent feature in the $2-10 \mathrm{keV}$ spectrum of a heavily obscured AGN, as in the case of IRAS $09104+4109\left(2 \times 10^{24} \lesssim N_{\mathrm{H}} \lesssim 10^{25} \mathrm{~cm}^{-2}\right.$, e.g. I01), is a strong Fe $\mathrm{K} \alpha$ emission line with an $E W_{\mathrm{K} \alpha} \gtrsim 1 \mathrm{keV}$ (Levenson et al. 2002; Guainazzi et al. 2005). This solid observational evidence matches well with the $E W$ value predicted by the theoretical calculations (e.g. Leahy \& Creighton 1993; Ghisellini et al. 1994; Matt et al. 1996). However, given the uncertainty in the reflection continuum flux due to the presence of the diffuse cluster emission, we also conservatively estimated the $E W_{\mathrm{K} \alpha}$ by using the lowest(highest) value in the $90 \%$ confidence interval measured for the normalization of the reflection component( $\mathrm{Fe} \mathrm{K} \alpha$ emission line) and vice versa. We obtained a range of $E W_{\mathrm{K} \alpha}$ values spanning from $\sim 165$ to $870 \mathrm{eV}$, which is marginally consistent with the $E W_{\mathrm{K} \alpha} \approx 1 \mathrm{keV}$ expected for a reflection-dominated scenario. As the continuum flux estimated for the obscured quasar with the reflection model $\left(F_{2-10}=4.68_{-1.08}^{+1.63} \times 10^{-13} \mathrm{erg} \mathrm{cm}^{-2} \mathrm{~s}^{-1}\right)$ is similar to the value measured with the spatially resolved Chandra spectrum $\left(F_{2-10} \approx 3.9 \times 10^{-13} \mathrm{erg} \mathrm{cm}^{-2} \mathrm{~s}^{-1}\right.$, e.g. I01), the marginal discrepancy in the $E W_{\mathrm{K} \alpha}$ values between the two observations arises from the different best-fit line flux intensity.
On the other hand, the $E W_{\mathrm{K} \alpha}=390_{-212}^{+380} \mathrm{eV}$ (note the large error bars) found in the case of the transmission model is in agreement with the expected $E W$ value of a $\mathrm{Fe} \mathrm{K} \alpha$ line transmitted through an absorbing screen with $N_{\mathrm{H}} \sim 5 \times 10^{23} \mathrm{~cm}^{-2}$ is ( 200-400 eV; e.g. Awaki et al. 1991; Leahy \& Creighton 1993; Ghisellini et al. 1994). A scenario with a Compton-thin absorber along the line of sight to the nucleus of IRAS 09104+4109 is therefore physically plausible. Nonetheless, the flux difference by a factor of $\sim 1.6-7.3$ between the 2003 XMM-Newton and 1998 BeppoSAX observation (a time interval of $3.5 \mathrm{yr}$ at the source frame after the correction for the time dilation due to the $z=0.442$ ) in the $20-30 \mathrm{keV}$ band is puzzling. In fact, given the observed power spectral densities of low black hole mass (i.e. $M_{\mathrm{BH}} \approx 10^{7} M_{\odot}$ ) Seyfert galaxies (Markowitz et al. 2003) and scaling linearly with the black hole mass (assuming $M_{\mathrm{BH}}=2.4 \times 10^{9} M_{\odot}$ for the nuclear black hole in IRAS $09104+4109^{2}$ ), flux variations of a factor of $\gtrsim 2$ in $3.5 \mathrm{yr}$ should be considered unlikely (e.g. Fiore et al. 1998). However, the major problem in the interpretation of this mismatch concerns the accuracy of BeppoSAX/PDS measurement. According to our analysis, the signal-to-noise ratio of these data is poor (only $\sim 2.5 \sigma$ between 15 and $50 \mathrm{keV}$ ). This implies that any conclusion based only on the PDS data about the Compton-thick or Compton-thin nature of the absorber in the nucleus of IRAS 09104+4109 could be misleading. The addition of a primary continuum power-law component modified by a Compton-thick absorber (i.e. similarly to the best-fit model proposed by F00 for the BeppoSAX broadband spectrum) might easily account for the above flux discrepancy, but, as stressed above, a scenario with a Compton-thick absorber for the EPIC spectrum is at odds with the inferred quite small value (i.e. $<1 \mathrm{keV}$ ) of the $\mathrm{Fe} \mathrm{K} \alpha E W$.

Given the large $1.3^{\circ}$ FWHM PDS field of view (FOV), PDS data might be affected by the contamination of very hard $\mathrm{X}$-ray sources possibly located outside the XMM-Newton and Chandra 15 arcmin radius FOVs. If a power-law source with $\Gamma=1.8$ is assumed, the $20-30 \mathrm{keV}$ flux measured for the PDS source $\left(F_{20-30} \sim 2.55 \times 10^{-12} \mathrm{erg} \mathrm{cm}^{-2} \mathrm{~s}^{-1}\right)$ is translated to $F_{2-10} \sim 7.5 \times 10^{-12} \mathrm{erg} \mathrm{cm}^{-2} \mathrm{~s}^{-1}$. According to the RXTE allsky slew survey $\log N-\log S$ function in the $2-10 \mathrm{keV}$ band (Revnivtsev et al. 2004), at this flux level 0.025 sources are expected in a $1.5 \mathrm{deg}^{2}$ area, which translates into a probability of $2.4 \%$. Moreover, using the NED and SIMBAD catalogs we found a probable contaminating source in the $\mathrm{H}_{2} \mathrm{O}$ maser galaxy NGC $2782(z=0.008)$, which is located at $\sim 50$ arcmin away from IRAS 09104+4109. The Chandra spectrum of this source (with a nuclear X-ray flux of a few times $10^{-13} \mathrm{erg} \mathrm{cm}^{-2} \mathrm{~s}^{-1}$ ) strongly suggests the presence of a heavily $\left(N_{\mathrm{H}}>10^{24} \mathrm{~cm}^{-2}\right)$ absorbed AGN (Zhang et al. 2006), whereby it is likely that NGC 2782 provides a sizable contribution to the $20-30 \mathrm{keV}$ flux measured with the PDS. Assuming a pure reflection model, we estimated a $F_{20-30} \sim 6 \times 10^{-13} \mathrm{erg} \mathrm{cm}^{-2} \mathrm{~s}^{-1}$, which must be considered a lower limit of the $20-30 \mathrm{keV}$ emission from NGC 2782, because of the likely presence of the nuclear continuum emerging after transmission through the absorber.

Alternatively, IRAS 09104+4109 could represent the first example of a "changing-look" quasar (Guainazzi et al. 2002; Matt et al. 2003, and reference therein) ever detected given that the XMM-Newton data are better explained by a transmissiondominated model with $N_{\mathrm{H}} \sim 5 \times 10^{23} \mathrm{~cm}^{-2}$ while, if the

\footnotetext{
${ }^{2}$ We estimated a mass of $2.4 \times 10^{9} M_{\odot}$ for the black hole in the nucleus of IRAS 09104+4109 using the measurement of the MgII line width from Hines \& Wills (1993), i.e. FWHM $=10000 \mathrm{~km} \mathrm{~s}^{-1}$, and the formula in Willott et al. (2003).
} 
PDS emission is entirely due to IRAS 09104+4109, the BeppoSAX data are consistent with a reflection-dominated spectral state. In fact, similar spectral transitions from a Comptonthick to a Compton-thin state (or vice-versa) have been observed, but only in Seyfert-like AGNs so far. This scenario implies a dramatic decrease (a factor of $\gtrsim 5-10$ ) in the line-of-sight absorbing column density during a timescale of 5 years and, in turn, suggests the presence of a largely inhomogeneous obscuring circumnuclear gas (Elvis et al. 2004; Elitzur \& Shlosman 2006). We estimated the line-of-sight crossing-time of an obscuring cloud in Keplerian motion around the central black hole of $2.4 \times 10^{9} M_{\odot}$ to explain the possible transition from a reflectiondominated to a transmission-dominated spectrum. We assumed a scenario similar to that described for NGC 1365 in Risaliti et al. (2007) (or NGC 3227, e.g. Lamer et al. 2003) where they found that the Compton-thick obscuring material responsible of the spectral transition is located in the broad line region (BLR) and the size of the X-ray emitting region is $\lesssim 100 \mathrm{R}_{\mathrm{G}}$. We estimated a distance of the BLR $R_{\mathrm{BLR}}=0.14 \mathrm{pc}$ using the empirical relation $R_{\mathrm{BLR}}-M_{\mathrm{BH}}$ in Kaspi et al. (2000). The redshiftcorrected crossing-time (Guainazzi et al. 2002) of a Keplerian cloud covering a region of size $100 R_{\mathrm{G}}$ around a black hole of $2.4 \times 10^{9} M_{\odot}$ is $\sim 1.7 \mathrm{yr}$. Such a value is therefore consistent with the $3.5 \mathrm{yr}$ (source frame) elapsed between the BeppoSAX and XMM-Newton observation.

Finally, we calculated a ratio $r_{\mathrm{X}, \mathrm{bol}} \equiv L_{2-10 \mathrm{keV}} / L_{\mathrm{bol}}=$ 0.016 using the $2-10 \mathrm{keV}$ luminosity of $L_{2-10 \mathrm{keV}}=7.95 \times$ $10^{44}$ erg $\mathrm{s}^{-1}$ measured for the transmission-scenario (see Table 1$)$, and a bolometric luminosity of $L_{\mathrm{bol}}\left(\approx L_{\mathrm{IR}}\right)=4.7 \times$ $10^{46} \mathrm{erg} \mathrm{s}^{-1}$, which is largely dominated by the obscured quasar (e.g. Hines et al. 1999; Spoon et al. 2007). We also derived the value of $r_{\mathrm{X}, \text { bol }}$ expected for IRAS 09104+4109 using the value of $v l_{v}(2500 \AA) / L_{\text {bol }}$ typical for quasars reported in Elvis et al. (1994), correcting the $L_{\text {bol }}$ value by a factor of $1 / 3$ as suggested by Fabian \& Iwasawa (1999) in order not to count twice the UV emission, and the spectral index $\alpha_{\mathrm{OX}}$ between $2500 \AA$ and $2 \mathrm{keV}$, defined as $\alpha_{\mathrm{OX}}=-0.384\left[l_{v}(2 \mathrm{keV}) / l_{v}(2500 \AA)\right]$ (e.g. Tananbaum et al. 1979). In particular, we used the relation $\alpha_{\mathrm{OX}}=0.137$ $\log \left(l_{v}(2500 \AA)\right)-2.638$ reported by Steffen et al. (2006). We converted from the monochromatic value of $v l_{v}(2 \mathrm{keV})$ to the $L_{2-10 \mathrm{keV}}$ value by multiplying by a factor of 1.61 , applying a photon index of $\Gamma=2$. We obtained that $r_{X, \text { bol }} \equiv L_{2-10 \mathrm{keV}} / L_{\mathrm{bol}}=$ $0.043 \times L_{\text {bol, } 45}^{-0.357}$ (with $L_{\mathrm{bol}, 45}=L_{\mathrm{bol}} / 10^{45} \mathrm{erg} \mathrm{s}^{-1}$ ). This implies that the expected value of $r_{\mathrm{X}, \text { bol }}$ for IRAS $09104+4109$ is 0.011 , which is close to the value of $r_{\mathrm{X}, \mathrm{bol}}=0.016$ measured using the $L_{2-10 \mathrm{keV}}$ derived for the transmission scenario. Since the observed luminosity of $L_{2-10 \mathrm{keV}}=2.05 \times 10^{44} \mathrm{erg} \mathrm{s}^{-1}$ in the reflection model should be just few percent of the intrinsic one (Comastri 2004; I01), this result lends further support to the hypothesis of a Compton-thin absorber along the line of sight to the nucleus of IRAS $09104+4109$.

\section{Summary}

The XMM-Newton observation of IRAS 09104+4109 suggests the possibility that the absorber along the line of sight to the nucleus of IRAS 09104+4109 is Compton-thin. If this is the case, it implies a scenario completely different from that reported for this type 2 quasar so far. It is worth stressing, however, that previous X-ray studies of IRAS $09104+4109$ inferred a reflection-dominated nature of its $2-10 \mathrm{keV}$ spectrum mainly on the basis of poor signal-to-noise $\sim 15-50 \mathrm{keV}$ data taken with the non-imaging BeppoSAX/PDS detector. Future imaging spectroscopy of IRAS $09104+4109$ performed in the 10$50 \mathrm{keV}$ range, say with Simbol-X or XEUS, is therefore needed to make definitive progress in measuring the exact continuum emission from the quasar and constraining the column density of the nuclear absorber. A deep Chandra observation of IRAS 09104+4109 would also be useful to accurately quantify the strength of the $\mathrm{Fe} \mathrm{K} \alpha$ emission line at $6.4 \mathrm{keV}$, which is a proxy for the Compton thickness of the absorber.

Acknowledgements. We thak the referee, Dr. K. Iwasawa, for careful reading and for many useful comments that helped us to improve the manuscript. We are grateful to G. Miniutti, R. Maiolino, S. Colafrancesco and G. Risaliti for helpful discussions. Based on observations obtained with XMM-Newton, an ESA science mission with instruments and contributions directly funded by ESA Member States and NASA. This research has made use of the NASA/IPAC Extragalactic Database (NED) which is operated by the Jet Propulsion Laboratory, California Institute of Technology, under contract with the National Aeronautics and Space Administration. We acknowledge financial contribution from contract ASI-INAF $\mathrm{I} / 023 / 05 / 0$.

\section{References}

Avni, Y. 1976, ApJ, 210, 642

Awaki, H., Koyama, K., Inoue, H., \& Halpern, J. P. 1991, PASJ, 43, 195 Comastri, A. 2004, in Supermassive Black Holes in the Distant Universe, ed. A. J. Barger (Kluwer Academic), 308, 245

Elitzur, M., \& Shlosman, I., 2006, ApJ, 648, L101

Elvis, M., Wilkes, B. J., McDowell, J. C., et al. 1994, ApJS, 95, 1

Elvis, M., Risaliti, G., Nicastro, F., et al. 2004, ApJ, 615, L25

Fabian, A. C., \& Crawford, C. S. 1995, MNRAS, 274, L63

Fabian, A. C., \& Iwasawa, K. 1999, MNRAS, 303, L34

Fiore, F., Laor, A., Elvis, M., Nicastro, F., \& Giallongo, E. 1998, ApJ, 503, 607 Franceschini, A., Bassani, L., Cappi, M., et al. 2000, A\&A, 353, 910 (F00) Ghisellini, G., Haardt, F., \& Matt, G. 1994, MNRAS, 267, 743

Guainazzi, M., Matt, G., Fiore, F., \& Perola, G.C. 2002, A\&A, 388, 787 Guainazzi, M., Matt, G., \& Perola, G. C. 2005, A\&A, 444, 119 Hines, D. C., \& Wills, B. J. 1993, ApJ, 415, 82

Hines, D. C., Schmidt, G. D., Wills, B. J., Smith, P. S., \& Sowinski, L. G. 1999, ApJ, 512, 145

Iwasawa, K., Fabian, A.C., \& Ettori, S. 2001, MNRAS, 321, L15, (I01)

Jansen, F., Lumb, D., Altieri, B., et al., 2001, A\&A, 365, L1

Jimenez-Bailon, E., Piconcelli, E., Guainazzi, M., et al. 2005, A\&A, 435, 449

Kallman, T. R., Palmieri, P., Bautista, A., Mendoza, C., \& Krolik, J. H. 2004, ApJ, 465, 994

Kaspi, V. M., Lackey, J. R., Mattox, J., et al. 2000, ApJ, 528, 445

Kleinmann, S. G., Hamilton, D., Keel, W. C., et al. 1988, ApJ, 328, 161

Lamer, G., Uttley, P., \& McHardy, I. M. 2003, MNRAS, 342, L41

Leahy, D. A., \& Creighton, J. 1993, MNRAS, 263, 314

Levenson, N. A., Krolik, J. H., Zycki, P. T., et al. 2002, ApJ, 573, L81

Markowitz, A., Edelson, R., \& Vaughan, S. 2003, ApJ, 598, 935

Matt, G., Guainazzi, M., \& Maiolino, R. 2003, MNRAS, 342, 422

Matt, G., Brandt, W. N., \& Fabian, A. C. 1996, MNRAS, 280, 823

Molendi, S., Bianchi, S., \& Matt, G. 2003, MNRAS, 343, L1

Murphy, E. M., Lockman, F. J., Laor, A., \& Elvis, M. 1996, ApJS, 105

Netzer, H., Lemze, D., Kaspi, S., et al. 2005, ApJ, 629, 739

Piconcelli, E., Jimenez-Bailon, E., Guainazzi, M., et al. 2004, MNRAS, 351, 161

Piconcelli, E., Jimenez-Bailon, E., Guainazzi, M., et al., 2005, A\&A, 432, 15

Reeves, J. N., et al. 2006, Astron. Nachr., 88, 789

Revnivtsev, M., Sazonov, S., Jahoda, K., \& Gilfanov, M. 2004, A\&A, 418, 927

Risaliti, G. 2002, A\&A, 386, 379

Risaliti, G., Elvis, M., Fabbiano, G., et al. 2007, ApJ, 659, L111

Spoon, H. W. W., Marshall, J. A., Houck, J. R., et al. 2007, ApJ, 654, L49

Steffen, A. T., Strateva, I., Brandt, W. N., et al. 2006, AJ, 131, 2826

Tananbaum, H., Avni, Y., Branduardi, G., et al. 1979, ApJ, 234, L9

Tran, H. D., Cohen, M.H., \& Villar-Martin, M. 2000, AJ, 120, 562

Willott, C. J., McLure, R. J., \& Jarvis, M. J. 2003, ApJ, 587, L15

Zhang, J. S., Henkel, C., Kadler, M., et al. 2006, A\&A, 450, 933 